\title{
A topological duality for posets
}

\author{
Luciano González ${ }^{1}$ and Ramon Jansana ${ }^{2}$ \\ 1 Facultad de Ciencias Exactas y Naturales, Universidad Nacional de La Pampa \\ 2 Departament de Lògica, Història i Filosofia de la Ciència, Universitat de Barcelona \\ luciano.javier.gonzalez@gmail.com \\ jansana@ub.edu
}

In $[2,3]$ Moshier and Jipsen develop a topological duality for lattices and extend it to lattice expansions with quasioperators. This duality builds on a topological duality they introduce for meet-semilattices. The ideas involved in obtaining it can be used to develop a duality for arbitrary posets. This is the purpose of our contribution. We will present the duality and apply it to prove by topological means the existence of the canonical extension of a poset, as defined in $[1]$.

A fundamental concept to obtain our duality is that of down-directed up-set, that we call a filter of the poset. The category Po of posets we consider has as objects the posets and as morphisms the order-preserving maps between posets such that the inverse image of a filter is a filter.

The dual spaces of the posets are the sober spaces $\langle X, \tau\rangle$ with the property that the compact open filters of $X$ w.r.t. the specialization order form a base for the topology $\tau$. We call these spaces P-spaces. The duals of the morphisms between posets of our category Po are the continuous functions between $P$ spaces with the property that the inverse image of a compact open filter is a compact open filter; we call such maps $F$-continuous maps. The $P$-spaces with the $F$-continuous maps form a category that we denote by PSp.

The categories Po and PSp are dually equivalent. Given a poset $P$, its dual space is obtained as follows. Let $\mathrm{Fi}(P)$ denote the set of the filters of $P$. We consider the Scott topology $\tau_{\mathrm{Fi}(P)}$ of the poset $\langle\mathrm{Fi}(P), \subseteq\rangle$. This space is sober. We denote the space by $X_{P}:=\left\langle\mathrm{Fi}(P), \tau_{\mathrm{Fi}(P)}\right\rangle$. The specialization order $\sqsubseteq$ of $X_{P}$ is the inclusion relation; that is, for $F, G \in \operatorname{Fi}(P), F \sqsubseteq G \Longleftrightarrow F \subseteq G$. For every $a \in P$ we define the set

$$
\varphi(a):=\{F \in \operatorname{Fi}(P): a \in F\} .
$$

The family $\{\varphi(a): a \in P\}$ is a base for the topology $\tau_{\mathrm{Fi}(P)}$ whose elements are exactly the compact open filters of $X_{P}$ (filter means here filter w.r.t. the specialization order). Accordingly we denote it by $\operatorname{KOF}\left(X_{P}\right)$. Of course, the map $\varphi$ is an order isomorphism between $P$ and $\operatorname{KOF}\left(X_{P}\right)$, ordered by the inclusion relation.

If $P$ and $Q$ are posets and $j: P \rightarrow Q$ is a map with the property that the inverse image of a filter of $Q$ is a filter of $P$, then the map $j^{-1}: \operatorname{Fi}(Q) \rightarrow \operatorname{Fi}(P)$ is a continuous map from $X_{Q}$ to $X_{P}$ with the property that the inverse image of a compact open filter is a compact open filter, i.e. it is an $F$-continuous map. 
Given a $P$-space, its dual poset is the set of its compact open filters ordered by inclusion. And if $X, Y$ are $P$-spaces and $f: X \rightarrow Y$ is $F$-continuous, then the map $f^{-1}: \operatorname{KOF}(Y) \rightarrow \operatorname{KOF}(X)$ is a morphism of the category Po.

The canonical extension of a poset $P$, as defined in [1], is (up to isomorphism) the lattice Fsat $\left(X_{P}\right)$ of the $F$-saturated sets of its dual space $X_{P}=$ $\left\langle\mathrm{Fi}(P), \tau_{\mathrm{Fi}(P)}\right\rangle$, where a subset of $\mathrm{Fi}(P)$ is $F$-saturated if it is the intersection of a family of open filters of $X_{P}$. Then an element $U \in \operatorname{Fsat}\left(X_{P}\right)$ is a closed element (in the sense of [1]) of the canonical extension if there is a filter $F$ of $P$ such that $U=\uparrow F$ in $\langle\operatorname{Fi}(P), \subseteq\rangle$, and it is an open element (in the sense of [1]) of the canonical extension if there exists an ideal $H$ of $P$ (i.e. an up-directed down-set) such that $U=\{G \in \operatorname{Fi}(P): G \cap H \neq \varnothing\}$.

\section{References}

1. Dunn, M., Gehrke, M., Palmigiano, A.: Canonical Extensions and Relational Completeness of Some Substructural Logics. The Journal of Symbolic Logic 70, 713-740 (2005)

2. Moshier, M.A., Jipsen, P.: Topological duality and lattice expansions, I: A topological construction of canonical extensions. Algebra Universalis 71, 109-126 (2014).

3. Moshier, M.A., Jipsen, P.: Topological duality and lattice expansions, II: Lattice expansions with quasioperators. Algebra Universalis 71, 221-234 (2014). 